\title{
Fire Toxicity Assessment: Comparison of Asphyxiant Yields from Laboratory and Large Scale Flaming Fires
}

\author{
ANNA A. STEC, T. RICHARD HULL, DAVID A. PURSER and JENNIFER A. PURSER \\ Centre for Fire and Hazard Science \\ University of Central Lancashire \\ Preston, Lancashire, PR1 2HE, UK
}

\begin{abstract}
In a fire, normally non-hazardous thermoplastic materials can produce lethal concentrations of toxic effluents in the smoke. The toxic product yields of the asphyxiants carbon monoxide (CO) and hydrogen cyanide $(\mathrm{HCN})$ from developed fires are typically 10 times greater than those generated in small-scale laboratory fire test apparatuses. Since most fire deaths, and most fire injuries, result from inhalation of toxic effluents, accurate replication is important for both fire hazard assessment and forensic fire investigation. The stoichiometric equivalence ratio allows the ventilation condition of flaming fires to be compared across different scales. The yields of $\mathrm{CO}$ and $\mathrm{HCN}$ from five laboratory-scale methods have been compared to large-scale data under the range of flaming fire conditions. Toxic product yield data from the smoke density chamber (SDC) (ISO 5659-2), the controlled atmosphere cone calorimeter (CACC) (based on ISO 5660), the fire propagation apparatus (FPA) (ASTM E 2058), the French railway test (NFX) (NF X 70100), and the steady state tube furnace (SSTF) (ISO/DIS 19700) are compared to published large-scale enclosure fire data (from a standard ISO 9705 room) for two polymers, polypropylene (PP) and polyamide 6.6 (PA 6.6). The results from the SSTF and FPA show the best agreement with those from the full and $1 / 3$ scale ISO room for both materials under a range of fire conditions. The CACC and SDC show reasonable agreement for well-ventilated burning, but fail to replicate the more hazardous under-ventilated fire conditions. The NFX generates data intermediate between the well-ventilated and under-ventilated fire conditions.
\end{abstract}

KEYWORDS: Controlled atmosphere cone calorimeter, ISO 5659, smoke density chamber, NF X 70-100, ISO 19700, fire propagation apparatus, ASTM E2058, laboratory-scale, fire, toxicity

\section{INFLUENCE OF FIRE CONDITIONS ON TOXICITY}

Although all fires may be regarded as unique, burning behaviour and toxic product yields depend most strongly on a few factors. While burning behaviour is dependent on physical aspects of the fire scenario, such as shape and orientation of the fuel, effluent toxicity is most dependent on material composition [1], temperature and oxygen concentration [2]. The generalised development of a fire has been recognised, and used to classify fire growth into stages, from smouldering combustion and early well-ventilated flaming, through to fully-developed under-ventilated flaming [3]. Although smouldering (oxidative pyrolysis) can generate toxicologically significant quantities of effluent (for example smouldering cotton, or polyurethane foam in a small enclosed space), the rate of reaction, and hence the amount of toxic species generated, is much lower, and typically only affects people within the enclosure. Well-ventilated fires are generally small, so extinguishment or escape is still feasible, and any fire effluent will accumulate just below the ceiling above head height. However, as they grow, and the upper layer descends, fires become ventilation controlled, and fires in enclosures such as buildings rapidly change from well-ventilated to underventilated. These fires are larger, and therefore produce greater volumes of effluent, affecting occupants over a much wider part of any building. In the laboratory assessment of fire hazard, for flammability, $21 \%$ oxygen is normally considered the worst case scenario, whereas for fire toxicity, under-ventilated flaming and oxygen concentrations below $10 \%$ are the worst case scenario. This arises because the yields of the major toxic products (particularly the asphyxiants carbon monoxide $(\mathrm{CO})$ and hydrogen cyanide $(\mathrm{HCN})$ ) will be much greater.

Almost all unwanted fires are diffusion flames, with inefficient mixing of fuel and oxygen. In contrast, combustion systems for energy release normally premix the fuel and oxidant, for example by allowing air access to the base of the flame, giving a much cleaner burn and higher combustion efficiency. The interior of large diffusion flames are always under-ventilated, because oxygen cannot penetrate the flame. For a particular material, at a certain flame height, these products of incomplete combustion escape through the FIRE SAFETY SCIENCE-PROCEEDINGS OF THE ELEVENTH INTERNATIONAL SYMPOSIUM pp. 404-418 
tip of the flame, and smoke appears at a characteristic "smoke point height", releasing unburnt hydrocarbons and carbon monoxide [4]. When flames penetrate the descending, oxygen-depleted, upper layer, combustion becomes increasingly inefficient. Thus, for any larger fire there will always be a significant yield of CO, hydrocarbons and smoke. Indeed data from large-scale fires $[5,6]$ in enclosures, such as a room, shows much higher levels of the asphyxiants $\mathrm{CO}$ and $\mathrm{HCN}$ under conditions of developed flaming. It is therefore essential to the assessment of toxic hazard from fire that each fire stage can be adequately replicated, and preferably the individual fire stages treated separately. The drive for internationally harmonised methods for assessment of combustion toxicity, through adoption of international standards, such as those of ISO, provides the framework for meaningful and appropriate use of toxicity data in the assessment of fire hazard. The purpose of this paper is to examine the validity of the input data.

\section{Assessment of Fire Toxicity}

Quantification of the yields of toxic effluents generated by burning materials is a necessary component of fire hazard assessment. This is usually achieved using a laboratory-scale test apparatus and procedure. The quantity and composition of the fire effluents is a function of the fuels involved and the fire conditions. These conditions affect the burning rate and the degree of oxidation of the effluent. In particular, the yields of combustion products from laboratory-scale methods depend on the fuel/air ratio, and whether the decomposition is flaming or non-flaming. High yields of $\mathrm{CO}$ and $\mathrm{HCN}$ from under-ventilated fires are responsible for most deaths, but this under-ventilated burning is the most difficult to create on a laboratory scale. A review of the extensive research predicting the carbon monoxide evolution from flames of simple hydrocarbons, has shown the importance of the equivalence ratio, $\phi$, in defining the fire condition, equation 1 [7]. The dependence of fire toxicity and toxic product yields on the ventilation conditions in fires emphasises the need for reliable replication of fire conditions.

$$
\phi=\frac{\text { actual fuel toair ratio }}{\text { stoichiometric fuel to air ratio }}
$$

An equivalence ratio of 0.5 represents a well-ventilated scenario, typical of an early growing fire, while a ratio of 2 corresponds to the under-ventilated stage responsible for high yields of toxic effluents. When $\phi=$ 1 the theoretical amount of air is available for complete combustion to carbon dioxide $\left(\mathrm{CO}_{2}\right)$ and water etc. The equivalence ratio approach has been refined and subdivided: "global" equivalence ratio has been distinguished from "plume" equivalence ratio to improve the prediction of yields of carbon monoxide and other products of incomplete combustion, where the effluent mixes with fresh air and forms a reactive hot layer. According to Pitts, this condition arises when the hot layer temperature exceeds $625^{\circ} \mathrm{C}$ [7]. In many fire tests the equivalence ratio can be determined directly: for fuels of known composition from the burning rate or mass loss rate and the air flow rate, provided all the air passes through the fire plume. Where the material is of unknown composition, the equivalence ratio can be deduced from the oxygen consumption, during well-ventilated burning. For a laboratory scale method to differentiate between the stages of a fire, an indicator of fire condition, such as the equivalence ratio or the combustion efficiency must be quantified so that the individual fire stages can be identified, and the product yields correlated to each stage.

Assessment of fire toxicity is currently focused on areas where escape is restricted, although most deaths and injuries actually occur in residential fires. Thus, most mass transport applications, such as aeroplanes, railway carriages, and passenger ships include requirements to quantify the fire toxicity of internal components. In most countries, there are no regulations covering the fire toxicity of building components, or for most road vehicles, including goods vehicles in tunnels. In China and Japan, there are specific restrictions on the use of materials with high fire toxicity in high risk applications such as tall buildings, while an increasing number of jurisdictions permit the alternative performance based design approaches to fire safety. As structures and means of transportation become larger and more complex, there is movement away from the more traditional methods of ensuring fire safety by prescriptive codes, towards fire risk assessments and engineering solutions, known as performance based design. Reliable rate of heat release, fire effluent toxicity and smoke generation data are all essential components of such an assessment. The general approach, described in ISO 13571 [8], is to ensure that the available safe escape time (ASET) before escape routes become obscured by smoke or filled with toxic gases, exceeds the required safe escape 
time (RSET). Various apparatus and protocols for quantifying fire effluent toxicity in different jurisdictions and industries have been critically reviewed [9]. The quantification of fire toxicity is being led by the ISO TC92 Subcommittee 3 - Fire Threat to People and the Environment, where the Steady State Tube Furnace (currently ISO/DIS 19700) is being developed specifically as the means of quantifying toxic product yields from burning materials on a laboratory scale. Meanwhile, the transport industries have tried to adopt the smoke density chamber (SDC) ISO 5659-2 [10], for quantification of toxic product yields [11, 12] using conventional or FTIR gas analysis, despite significant problems of reproducibility. It has been suggested that the reproducibility problems arose from the single point measurement (the tip of the probe may be in the centre of the plume, below it, or if mixing is more efficient, the upper layer may be recirculated through the flame), or the timing of the effluent sampling may cause instabilities (for example an initial proposal to sample after 8 minutes was replaced by a proposal to sample when the smoke density reached its maximum). The reproducibility of a revised protocol is being investigated based on continuous sampling of the fire effluent. In addition, the European rail standard [12] also specifies use of the French Railway test (NF X 70-100) [13] for smaller components. Although at an earlier stage of development, the controlled atmosphere cone calorimeter (CACC) is being considered within ISO as an apparatus for quantifying heat release rates from under-ventilated flaming, and has been proposed as a potential candidate for quantifying toxic products yields from different fire conditions. The fire propagation apparatus (FPA) was developed by the US insurance company Factory Mutual (now FM Global), but has been standardised for heat release measurement [14], but not for quantification of toxic fire effluents.

\section{Laboratory-Scale Methods for Generation of Fire Effluents}

For input into models of fire toxicity, laboratory-scale methods used for generation of toxic fire effluents should be capable of reproducing individual fire stages or combustion conditions. Full-scale fires simultaneously involve different fire stages in different places, which are changing with time. However, bench-scale methods which allow the combustion conditions to change during the test are much more difficult to relate to full-scale fires, because the duration of each condition is unknown, and the behaviour of fires changes on scale-up. Most laboratory-scale methods have non-constant combustion conditions, such as those in closed chambers exposed to a constant source of heat, including the smoke density chamber (SDC) (ISO 5659-2) [10], and static tube furnace tests, such as the NF X 70-100 (NFX) [13]. Those with constant combustion conditions are more suited to producing data suitable for comparison and modelling: the steady state tube furnace (SSTF) (ISO/DIS 19700) [15] has been specifically designed to achieve this. Intermediate between these two approaches are those that can produce quasi-steady combustion conditions, such as the cone calorimeter (ISO 5660-1) [16] with non-standardised controlled atmosphere attachment (CACC), and the fire propagation apparatus (FPA) (ISO 12136) [14]. The difficulty of replicating the conditions of fully developed under-ventilated flaming on a laboratory-scale is caused by several practical problems. $\phi$ depends on the mass loss rate of the specimen and the available air; for most methods one or both are unknown; $\phi$ will be increased by an unknown factor if products are recirculated into the flame zone. Apparatuses where $\phi$ changes rapidly allow little time for sampling and measurement of mass loss and effluent composition, with resultant errors and uncertainties. Progressive changes in the composition of a static specimen (for example due to char formation) provide additional complexity. The reactions of under-ventilated flaming occur in both the flame zone and in the hot upper layer. Only the SSTF has a heated reaction zone which replicates the hot layer. The applied heat flux must be large enough for burning to continue at oxygen concentrations as low as $5 \%$. In some laboratory-scale apparatuses the heat flux is constant, and often insufficient to sustain flaming at such low oxygen concentrations; further, an unknown quantity of fresh air bypasses the fire plume, so the ventilation condition remains undefined. Some fire models, such as the cone calorimeter, fire propagation apparatus and smoke density chamber use the temperature of the radiant heater to preselect the radiant heat flux, and then check this using a radiant heat flux meter. Others, such as the NF X 70-100, and the ISO 19700 SSTF use the furnace temperature setting to ensure a consistent radiant heat flux. The radiant heat flux in the ISO 19700 apparatus has been measured [17] and is $40 \mathrm{~kW} \mathrm{~m}^{-2}$ in the centre of the furnace at $650^{\circ} \mathrm{C}$ and $78 \mathrm{~kW}$ $\mathrm{m}^{-2}$ at $825^{\circ} \mathrm{C}$.This paper describes each method briefly, and then presents experimental data comparing these different methods, and relates it to large-scale test data. 
The Smoke density chamber (ISO 5659-2)[10], shown in Figure 1, is the most widely used fire-test apparatus, stipulated in smoke regulations in most countries of the developed world. Its widespread availability has encouraged its adaptation to toxic gas generation and assessment. The standard specifies four test conditions which it is assumed are representative of different fire scenarios, by varying the heat flux in the presence or absence of a pilot ignition source. Of the conditions specified here: $25 \mathrm{~kW} \mathrm{~m}^{-2}$ without piloted ignition (presumably corresponding to oxidative pyrolysis); $25 \mathrm{~kW} \mathrm{~m}^{-2}$ with piloted ignition (corresponding to early, well-ventilated flaming); and $50 \mathrm{~kW} \mathrm{~m}^{-2}$ without piloted ignition (corresponding to developed, under-ventilated flaming). The fourth condition $\left(50 \mathrm{~kW} \mathrm{~m}{ }^{-2}\right.$ with piloted ignition) was introduced in the 2012 version, after the experimental work was completed. The sample is a $75 \mathrm{x} 75 \mathrm{~mm}$ square solid sheet and the standard for smoke measurement states that the results are only valid at the thickness tested (typically $1-4 \mathrm{~mm}$ ). For a fixed chamber volume $\left(0.51 \mathrm{~m}^{3}\right)$, assuming complete combustion, the sample thickness will dictate the ventilation condition, thus a thin sample will burn under well-ventilated conditions with minimum toxic products, while a thicker sample would be expected to produce a high yield of $\mathrm{CO}$ and other products of incomplete combustion. The protocol has been modified as a toxicity test by the mass transport industries, in the aircraft [18], maritime [11], and railway tests [12]. Some of these methods attempt to address the transition through the fire stages by monitoring the formation of toxic gases as a function of time, as the oxygen concentration falls, and the fire condition changes from well-ventilated to under-ventilated. However, unlike a real fire, the heat flux remains constant, and so when the oxygen concentration falls, the flame may be extinguished.

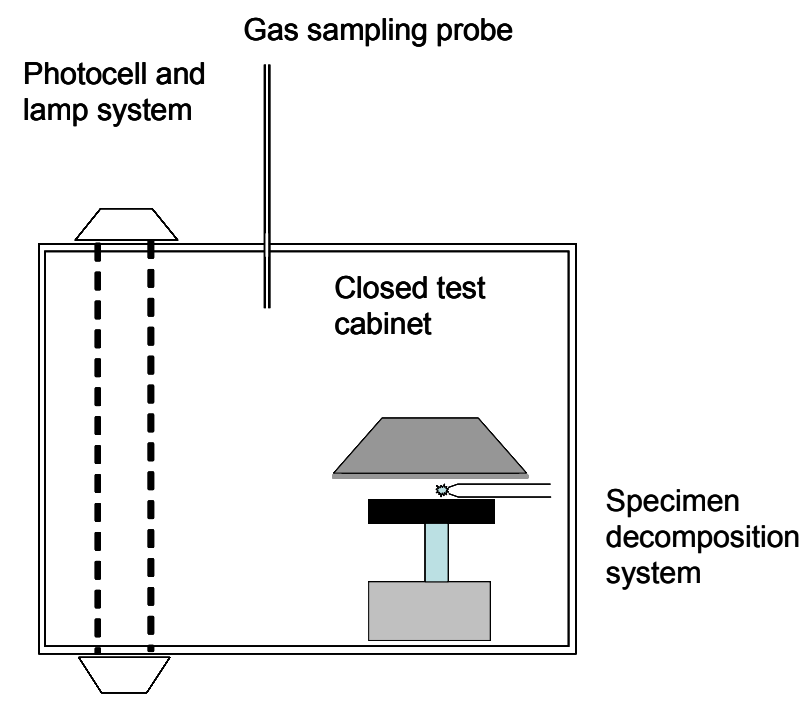

Figure 1 The smoke density chamber (ISO 5659-2) showing a sampling probe for fire smoke toxicity.

\section{The Controlled Atmosphere Cone Calorimeter (CACC)}

The cone calorimeter (ISO 5660) [16] is probably the most widely used apparatus for measurement of flammability properties such as ignitability and heat release rate [19]. It can be used for testing samples $100 \times 100 \mathrm{~mm}$ and up to $25 \mathrm{~mm}$ thick, in both the horizontal and vertical orientation. The open cone calorimeter replicates the early well-ventilated stage of flaming where a fire would be too small to produce enough toxicants to cause harm except in very small enclosures. However, a non-standard modification of the apparatus has been described, enclosing the fire model in a controlled ventilation chamber, in an attempt to replicate oxygen-depleted conditions. In this modification, the controlled atmosphere cone calorimeter (CACC) [20], shown in Figure 2, the fire model is enclosed in a heat resistant glass chamber (400 mm high with $300 \times 300 \mathrm{~mm}$ base) so that the air flow may be controlled. These modified cone calorimeters do not follow a particular standard, and are still under development. In the tests described 
here, the effluent continued to burn as it emerged from the chamber, (secondary flaming in figure 4) ultimately giving well-ventilated flaming. In others, under reduced oxygen concentrations, the fuel lifts from the surface, but ignition does not occur [21]. Hietaniemi [22] used the controlled atmosphere cone calorimeter, but argues correctly, we believe, that an instantaneous "effective" global equivalence ratio $\phi_{\text {eff }}$, should be used, rather than an averaged local equivalence ratio, based on the oxygen supply to the chamber, because, in some experiments, substantial secondary flaming occurred outside the test chamber, such that the amount of oxygen available to combustion exceeded the amount that was fed to the enclosed chamber.

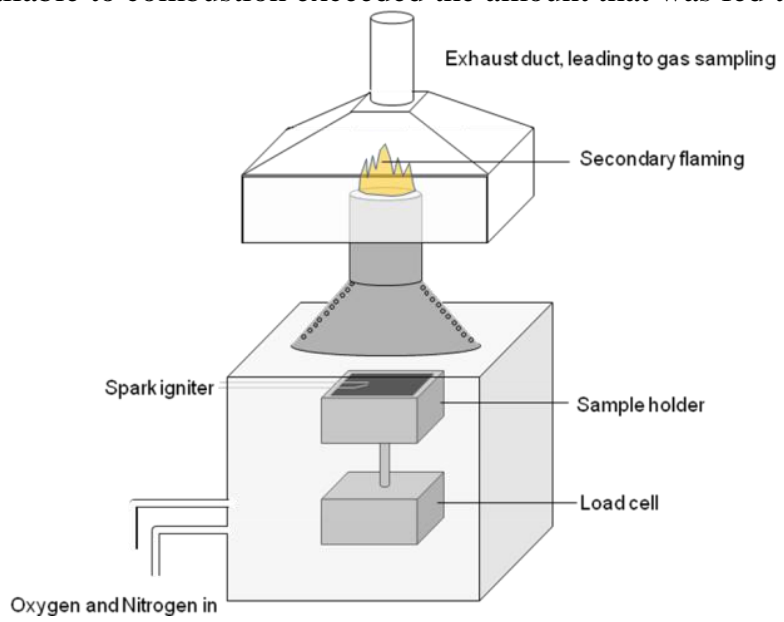

Figure 2 The controlled atmosphere cone calorimeter

\section{The Fire Propagation Apparatus (FPA)}

The fire propagation apparatus [14] (Figure 3) is similar in principle to the cone calorimeter but the fire zone is contained within a $172 \mathrm{~mm}$ diameter circular vertical silica tube, allowing better mixing of the fuel oxidant and avoiding contact with the heaters, which are outside the tube. Horizontal test specimens, typically $100 \mathrm{~mm}$ square and up to $25 \mathrm{~mm}$ thick, or vertical specimens $100 \mathrm{~mm}$ wide, $305 \mathrm{~mm}$ high and up to $25 \mathrm{~mm}$ thick, are exposed to thermal radiation and a pilot flame. The effluent flows through an instrumented duct and the rate of heat release is determined from the $\mathrm{CO}_{2}$ and $\mathrm{CO}$ generation rates. The effluent yields can be directly related to individual fire stages through the equivalence ratio. It has been used to quantify the generation of toxic products under different ventilation conditions, and data has been published describing $\mathrm{CO}$ and hydrocarbon yields from various polymers [23].

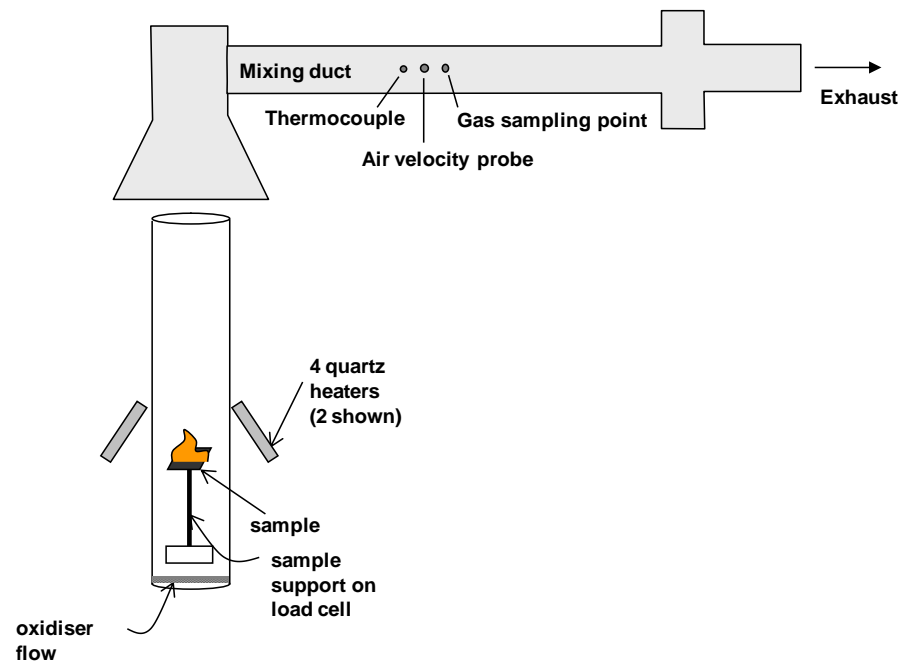

Figure 3 The fire propagation apparatus (ISO 12136) 


\section{The Non-dynamic Tube Furnace (NFX)}

In the standard Tube furnace thermal degradation method [13], the static specimen is thermally decomposed in a furnace with a metered air flow, which drives the effluent to the sampling system or gas measurement devices. Mass loss is obtained by weighing the sample before and after test. Determination of the equivalence ratio is not possible unless the rate of pyrolysis is known, for example from on-line analysis. Since different materials pyrolyse at different rates and have different stoichiometric oxygen requirements, using the same air flow will result in burning at equivalence ratios that vary with furnace temperature and material.

The NF X 70-100 method (NFX) shown in Figure 4 was developed to quantify the toxicity of materials and products used in railway vehicles. The static tube furnace uses 1 gram (or $0.1 \mathrm{~g}$ for low density materials) of sample in a crucible, inserted into the middle of a silica furnace tube of diameter $44 \mathrm{~mm}$ and thermally decomposed in air flowing at 2 litres $\mathrm{min}^{-1}$. Furnace temperatures of 400,600 and $800^{\circ} \mathrm{C}$ are used. Although used as a standard test, without correlation of the conditions to particular fire stages, it would appear that a temperature of $400^{\circ} \mathrm{C}$ corresponds to pyrolysis without ignition; a temperature of $600^{\circ} \mathrm{C}$ autoignites the sample with a fairly slow rate of burning which may correspond to well-ventilated flaming; a temperature of $800^{\circ} \mathrm{C}$ pyrolyses the sample so quickly that the fire condition becomes under-ventilated. In the current work, the three furnace temperatures have been considered to represent oxidative pyrolysis, well-ventilated and under-ventilated conditions respectively.

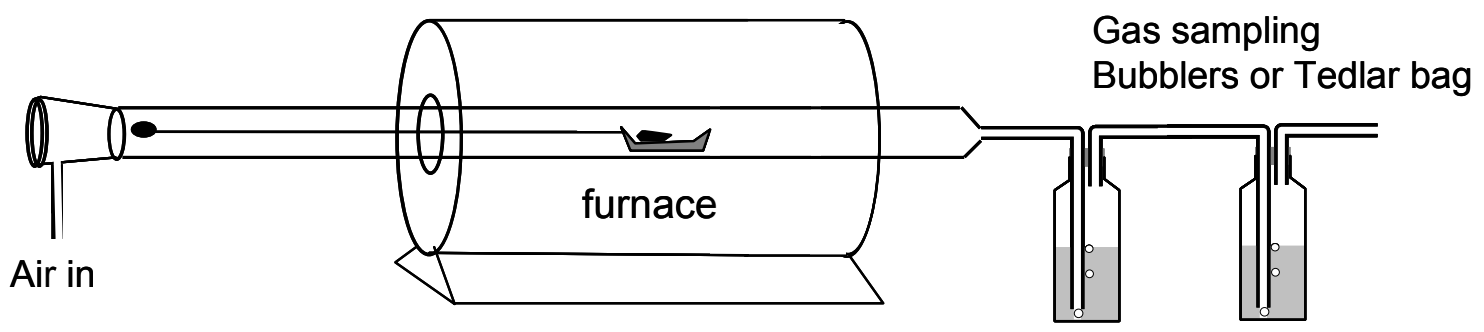

Figure 4 The non-dynamic tube furnace test, NF X 70-100

The method is easy to use, uses simple equipment with specified operating conditions of temperature and air flow. The greatest sensitivity in the test, leading to problems of repeatability and reproducibility, occur when the sample is close to ignition and in some cases flaming combustion occurs, while in other cases it does not. This makes the results very susceptible to differences in furnace design [24]. As part of EN TS 45545 , this method is increasingly used for fire toxicity testing of materials used for railway vehicles. The lack of requirement for flaming to be observed leaves the assignation of fire stages to be assumed. A practical problem is that several replicate test runs are often needed to obtain sufficient volume of sample for complete gas analysis.

\section{The Steady State Tube Furnace (SSTF)}

The steady state tube furnace [15], shown in Figure 5, feeds the sample (typically around $25 \mathrm{~g}$ of pellets or granules) into its hot zone at a fixed rate, under a controlled air supply, inside a horizontal silica tube of diameter $48 \mathrm{~mm}$, allowing adequate mixing of fuel and oxidant. It forces combustion by driving the sample into a furnace of increasing heat flux at a fixed rate, so that, by running several tests with the same material with different ventilation conditions, each fire stage can be replicated by steady state burning. The products generated in the flame zone then pass through the heated furnace tube, maintaining a high temperature, as in the upper layer of a compartment fire. The toxic product yields may be quantified from the gas concentrations and mass feed rate during the steady state burn period, after the initial peak.

This method has been designed to generate data for input to fire hazard assessments, using the methodology in ISO 13344 [25] and ISO 13571 [8], particularly in relation to the ISO fire stages [3]. The sample is 
spread evenly in a long silica boat over a length of $800 \mathrm{~mm}$ and fed into a tube furnace at a typical rate of $1 \mathrm{~g} \mathrm{~min}^{-1}$ with flowing air. Secondary air is added in a mixing chamber to give a total gas flow of 50 $\mathrm{L} \min ^{-1}$.

Thermocouple

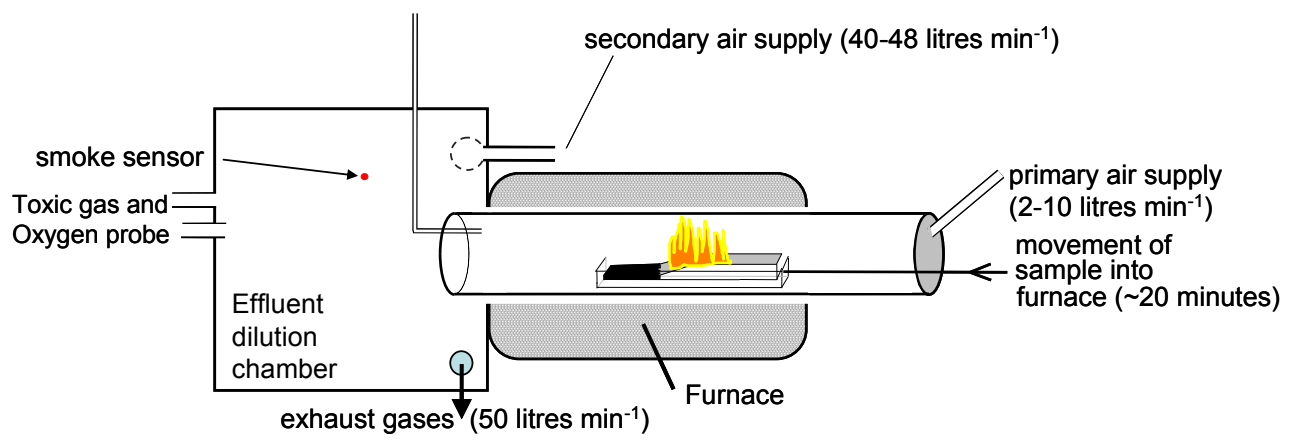

Figure 5 The steady state tube furnace apparatus, ISO 19700.

\section{Large Scale Fire Tests}

Unfortunately there is only a limited amount of suitable large scale data available where the fire condition is both known (in terms of equivalence ratio), and flaming was under-ventilated. One of the best data sets comes from the TOXFIRE project [22, 26, 27, 28], where a phi meter [29] was used to establish the fire condition inside a lightweight concrete room with dimensions in accordance with the ISO 9705 room test [30]. The ISO 9705 room has one opening, $0.8 \mathrm{~m}$ wide, $2.0 \mathrm{~m}$ high, centrally located at one end. The ventilation conditions inside the room were controlled by sealing the lower part of the opening with slabs of non-combustible fibreboard, giving a reduced opening of height $0.89,0.68,0.56$ and $0.45 \mathrm{~m}$, to create under-ventilated conditions. The fuel was put into square pans of $1.2 \mathrm{~m}^{2}$ for polypropylene (PP) and $1.4 \mathrm{~m}^{2}$ for polyamide 6.6 (PA 6.6). Four tests were carried out, burning $60 \mathrm{~kg}$ of PP and between $55-75 \mathrm{~kg}$ of PA 6.6, using a load cell to measure the mass loss. A phi meter determines the equivalence ratio of the fire by adding oxygen to the fire effluent, passing the mixture over a catalyst at $900^{\circ} \mathrm{C}$ then measuring the resulting oxygen concentration. Corresponding data have been reported for the different fire conditions using the bench-scale methods described above.

\section{CORRESPONDENCE OF YIELDS FROM LABORATORY SCALE AND LARGE SCALE FIRE TESTS}

\section{Methods which can be related to equivalence ratio}

Quantifying fire toxicity on a laboratory-scale is only meaningful if it relates to large scale behaviour. Steady state and quasi steady state methods allow the equivalence ratio to be determined. If large scale fire toxicity test data has also been recorded as a function of equivalence ratio, direct comparison is possible. For both PP and PA 6.6 burning under the conditions described, negligible residue remains, and therefore the mass charge, and mass loss yields will be indistinguishable. Figure 6 shows a comparison for the CO yield from PP as a function of equivalence ratio, using controlled atmosphere cone calorimeter data published as part of the TOXFIRE project [22], steady state tube furnace data [31], and data from the fire propagation apparatus (actually reported for LDPE which is isomeric with PP) [23], compared to the large scale data [26]. It is apparent from all tests that the inherent variation in burning behaviour gives much larger scatter in under-ventilated conditions than in well-ventilated conditions. In general, this shows a significant increase in the $\mathrm{CO}$ yield as the fire moves from well-ventilated to under-ventilated. Yields from the controlled atmosphere cone calorimeter are shown as average values as a function of equivalence ratio $(\phi)$ [26] and corrected values of the equivalence ratio, $\left(\phi_{\text {eff }}\right)$ where the oxygen depletion which resulted from flaming occurring outside the enclosure, above the cone heater, detailed in reference [22], was taken into account in the equivalence ratio calculation. With a single exception, the controlled atmosphere cone calorimeter failed to replicate the higher $\mathrm{CO}$ yields associated with large scale behaviour. 


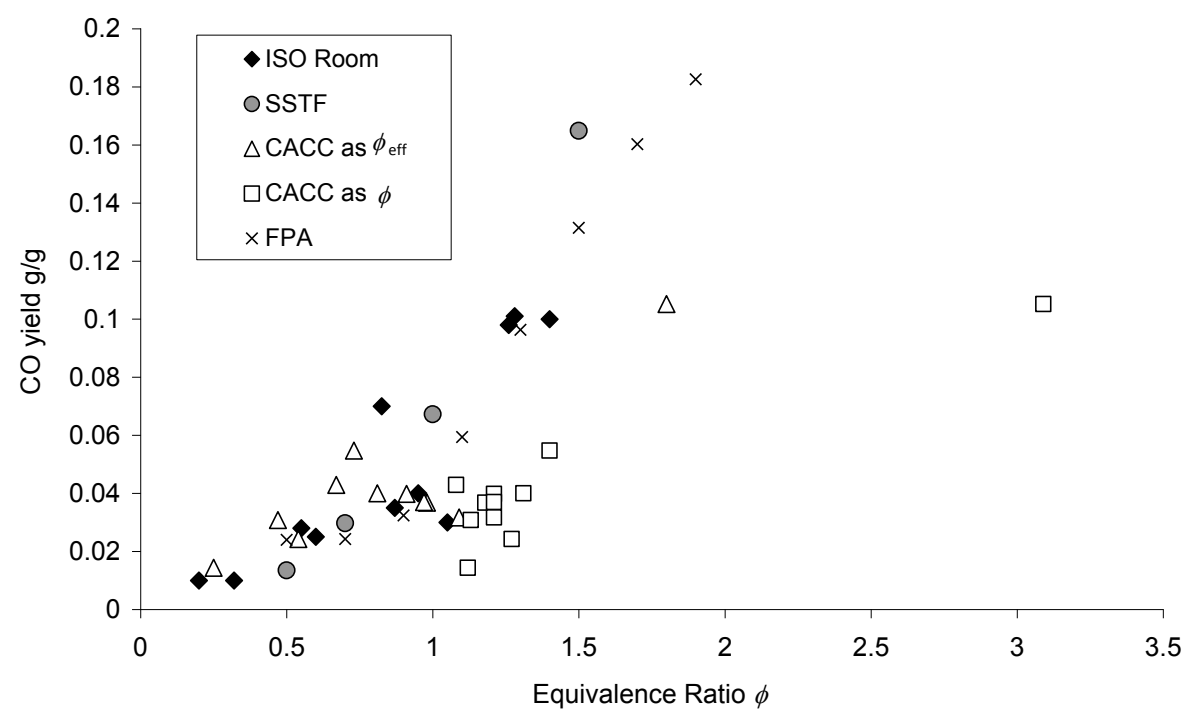

Figure 6 Comparison of CO yield for polypropylene for large scale (ISO Room), steady state tube furnace, fire propagation apparatus and controlled atmosphere cone calorimeter, as a function of equivalence ratio $\phi$.

Figure 6 shows acceptable agreement between the ISO room data and the steady state tube furnace, the controlled atmosphere cone calorimeter, and fire propagation apparatus data which actually generated greater extremities of well- and under-ventilated burning than occurred in the ISO room. However, in the results from the controlled atmosphere cone calorimeter, there is only a single data point (at $\phi=3.2$ or $\phi_{\text {eff }}$ $=1.8$ ) which shows the higher yields of carbon monoxide associated with under-ventilated combustion, suggesting that it is not easy to replicate this condition in the CACC. Data for the controlled atmosphere cone calorimeter generally shows lower $\mathrm{CO}$ yields in under-ventilated combustion, and after the correction for combustion outside the chamber, the apparatus is unable to replicate the more toxic conditions of underventilated burning responsible for most fire deaths, and therefore has limited value as a tool for assessment of fire toxicity.

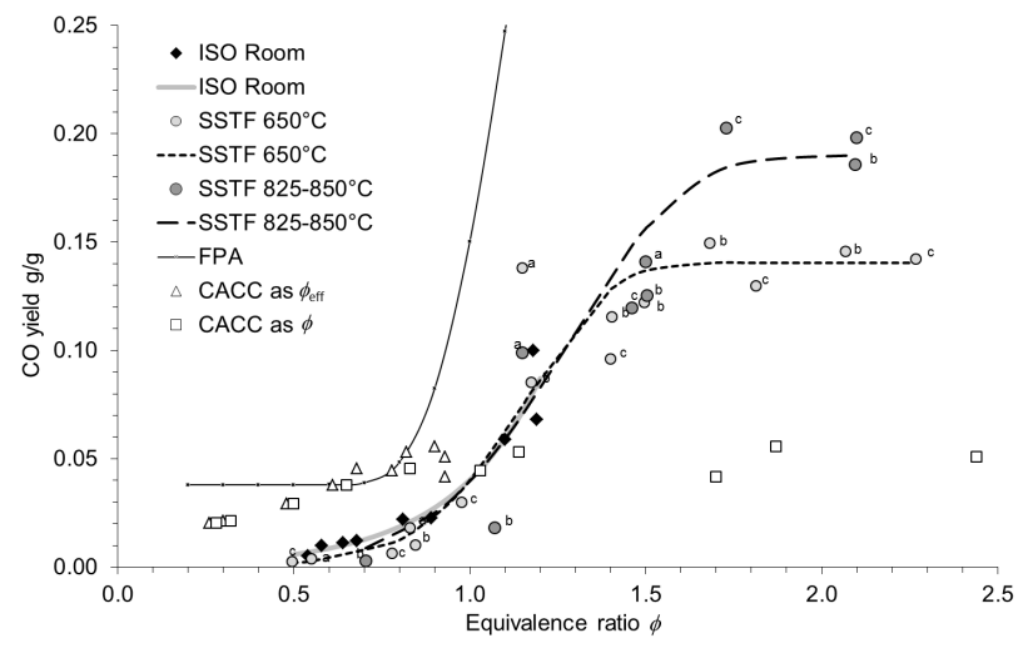

Figure 7 Comparison of $\mathrm{CO}$ yield for aliphatic polyamides from steady state tube furnace, controlled atmosphere cone calorimeter, fire propagation apparatus, with ISO Room, as a function of equivalence ratio $\phi$. Letters indicate data source and material for SSTF: a) polyamide 6.6 [32], b) polyamide 6.6 [2], c) polyamide $6[33]$. 
Figure 7 compares the published $\mathrm{CO}$ yields from the large scale ISO room tests, for PA 6.6 under a range of ventilation conditions with those from the controlled atmosphere cone calorimeter [22], steady state tube furnace [31], and the fire propagation apparatus [23]. The SSTF data include results from four data sets: a) Blomqvist [32], b) Stec [2] and c) Purser [33]. Of the three SSTF data sets, a) was obtained using the same batch of polyamide 6.6 as was used for the ISO 9705 Room in the TOXFIRE project; b) was polyamide 6.6, obtained from a different source; c) was for polyamide 6 (which has identical chemical composition and a slightly different structural formula to PA 6.6). Again, this shows a significant increase in the CO yield as the fire moves from well-ventilated to under-ventilated, accompanied by an increase in scatter, using all methods. CO yields in the SSTF were found to be temperature sensitive, with somewhat higher yields at $825-850^{\circ} \mathrm{C}$ than at $650^{\circ} \mathrm{C}$. During the ISO room tests at the higher equivalence ratios the upper layer temperatures were $750-1000^{\circ} \mathrm{C}$, corresponding more with the higher temperature SSTF results. The SSTF results are very similar from all three aliphatic polyamide samples. It is notable that the controlled atmosphere cone calorimeter results, based on the averaged local equivalence ratio $\phi$, reported by Hietaniemi [22] show higher CO yields in well-ventilated conditions and, crucially, lower CO yields in under-ventilated conditions. Again, the data have been corrected by calculation of the effective equivalence ratio $\phi_{\text {eff }}$ which aligns the yields to the well-ventilated stages of the large scale data [22].

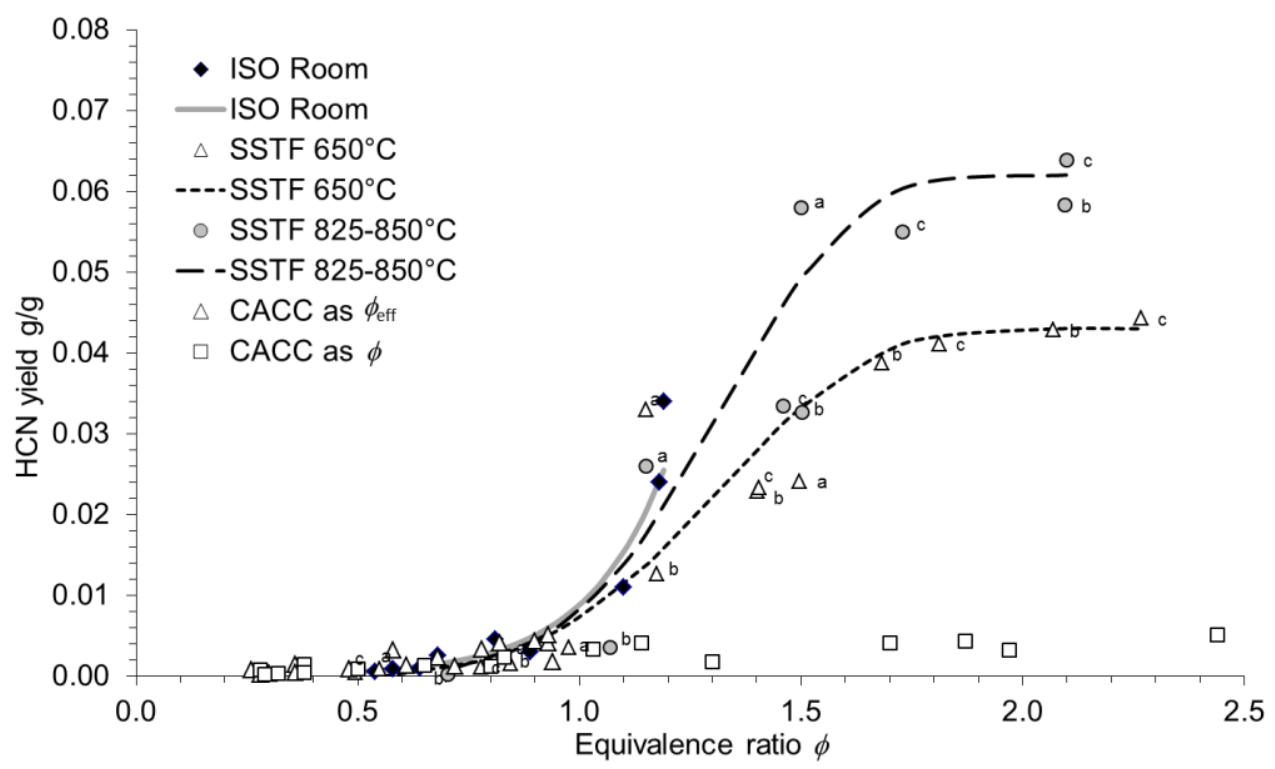

Figure 8 Comparison of HCN yield for aliphatic polyamides from steady state tube furnace and controlled atmosphere cone calorimeter with ISO Room, as a function of equivalence ratio $\phi$. Letters indicate data source and material for SSTF: a) polyamide 6.6 [32], b) polyamide 6.6 [2], c) polyamide 6 [33].

Figure 8 shows the same ISO room experiments compared for HCN yield to the steady state tube furnace, and the controlled atmosphere cone calorimeter. Again this shows good agreement between SSTF and large scale, alongside the sensitivity of $\mathrm{HCN}$ yields from polyamides to the furnace temperature in underventilated conditions. This illustrates the failure of the controlled atmosphere cone calorimeter to replicate the conditions of under-ventilated combustion even more dramatically. While the controlled atmosphere cone calorimeter data fit well with the large scale data up to $\phi=1$, they do not replicate the higher $\mathrm{CO}$ and HCN yields for under-ventilated conditions where $\phi>1$. Unfortunately Tewarson has not reported a similar correlation for HCN yield from PA 6.6 from the fire propagation apparatus. It is apparent that the controlled atmosphere cone calorimeter also fails to replicate the higher yields of the other major asphyxiant, hydrogen cyanide in under-ventilated fires. Indeed, the $\sim 50$ fold increase in HCN yield as the fire condition changes from well-ventilated to under-ventilated is not seen using the CACC. Again, correcting the data to $\phi_{\text {eff }}$ merely compresses it to below an equivalence ratio of 1.0 . 
Thus the steady state tube furnace and fire propagation apparatus can adequately replicate large scale burning, but the controlled atmosphere calorimeter cannot. Two recent reports of the use of the CACC for assessment of fire toxicity describe experimental programs investigating a range of fuels. In one, 6 materials, including PVC, polyisocyanurate foam, and a glass reinforced polyester containing brominated flame retardant are described [34], but the only results reported are the $\mathrm{HCl}$ yields. In the other paper [35], some carbon monoxide yields are reported for the burning of an undefined "sandwich composite material" which shows higher $\mathrm{CO}$ yields (up to $0.07 \mathrm{~g} / \mathrm{g}$ ) in well-ventilated conditions, (the oxygen concentration was $21 \%$ and $14 \%)$, and much lower $(0.03 \mathrm{~g} / \mathrm{g})$ for oxygen concentrations of 0,5 and $9 \%$. Based on these results, the authors conclude that "no gas phase oxidation can occur below $10 \% \quad \mathrm{O}_{2}$ : the formation of $\mathrm{CO}$ tends to decrease." This is in direct contradiction of the observations from large scale fires where CO yields above $0.2 \mathrm{~g} / \mathrm{g}$ are common in oxygen concentrations below $10 \%$, presumably because burning in the CACC cannot be sustained at the applied heat flux. Indeed upper layer oxygen concentrations as low as $1.2 \% \mathrm{O}_{2}$ have been measured [36]. It appears that there is a significant body of work necessary before the CACC can replicate the higher yields of toxic products associated with under-ventilated flaming.

\section{Methods where equivalence ratio is indeterminate}

For non-constant combustion conditions, although the equivalence ratio cannot be obtained, yields of major toxic products are available, and loosely assigned to fire condition. Steady state tube furnace (SSTF), fire propagation apparatus (FPA), and large scale ISO Room fire test data, have been compared to yields from the static tube furnace (NFX) and smoke density chamber (SDC) tests for LDPE/PP and PA 6.6[37].

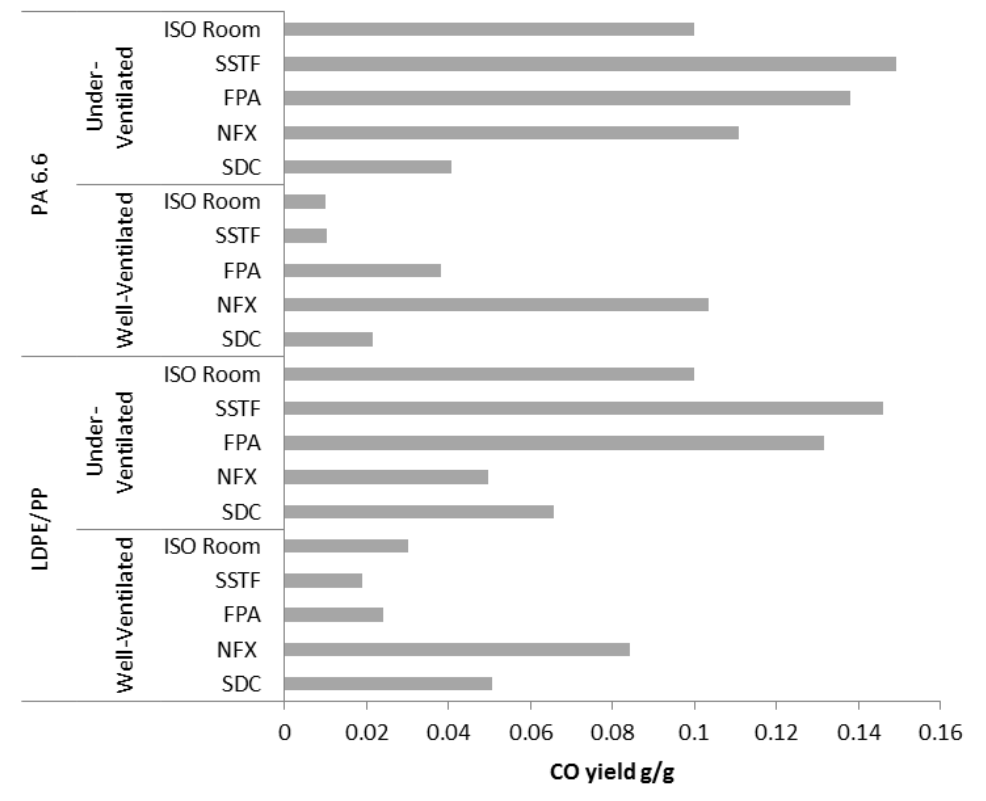

Figure $9 \mathrm{CO}$ yield in different laboratory scale tests and fire conditions.

Figure 9 compares the CO yield for LDPE/PP and PA 6.6 in the ISO Room, SSTF and FPA in wellventilated and under-ventilated conditions to those in the NFX and SDC tests. It shows very high CO yields for the well-ventilated conditions for LDPE and PA 6.6 for the NFX test, and better agreement of $\mathrm{CO}$ yields for the SDC. For the under-ventilated condition, for LDPE both the NFX and SDC tests underestimate the CO yield, while for PA 6.6, the NFX shows good agreement for CO, while the SDC shows the poorest agreement. 


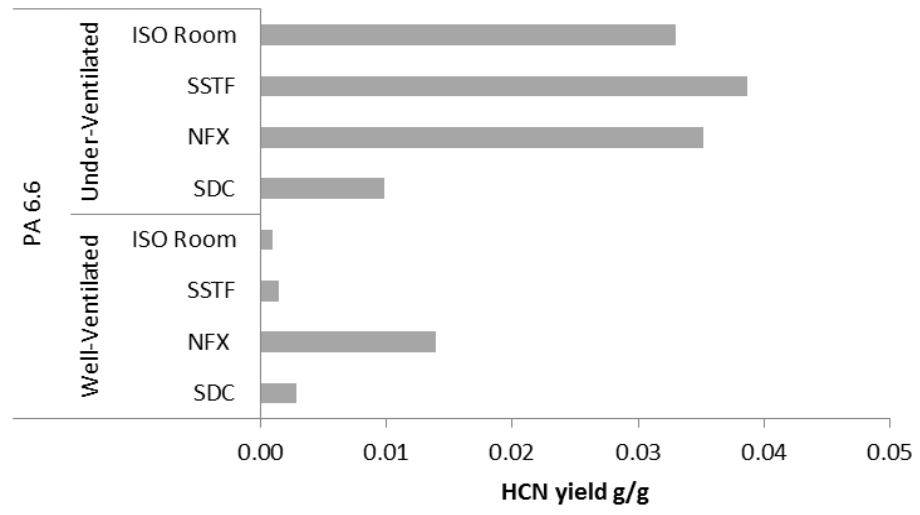

Figure $10 \mathrm{HCN}$ yield in different laboratory scale tests and fire conditions.

Figure 10 compares the HCN yield for PA 6.6 in the ISO Room and SSTF in well-ventilated and underventilated conditions to those in the NFX and SDC tests. This also shows high HCN yields for wellventilated conditions in the NFX test, but lower HCN yields for the SDC. In contrast, in under-ventilated conditions, the NFX test shows good agreement with the SSTF, while the SDC fails to replicate the high HCN yields associated with under-ventilated flaming.

Overall, the NFX test overestimates the yields from well-ventilated fires, but underestimates the yields from under-ventilated fires; the SDC replicates well-ventilated flaming, but not under-ventilated fire conditions.

\section{Attempted replication of under-ventilated flaming in the smoke chamber}

The results described in the preceding section led to a further investigation into the capability of the smoke chamber to replicate under-ventilated fires by burning different thicknesses of LDPE [37]. The equivalence ratio was calculated assuming complete combustion, and Tewarson's equation [23] was used to predict the

$\mathrm{CO}$ yield, and these predictions are compared to the actual results shown in

Table 1. When the tests were conducted under the most severe regime in the smoke chamber protocol, at 50 $\mathrm{kW} \mathrm{m} \mathrm{m}^{-2}$, as the atmosphere became oxygen depleted, instead of under-ventilated burning, the samples extinguished. Although the thicker samples were 10 and $15 \mathrm{~mm}$, only the upper $5-7 \mathrm{~mm}$ actually burnt, giving equivalence ratio and $\mathrm{CO}$ yields corresponding to well-ventilated flaming. Thus, like the controlled atmosphere cone calorimeter, it was not possible to replicate under-ventilated burning in the smoke chamber.

Table 1 Predicted and actual effect of sample thickness on CO yields in SDC.

\begin{tabular}{|c|c|c|c|c|c|c|c|}
\hline & \multicolumn{3}{|c|}{ Predicted for 100\% mass loss } & \multicolumn{4}{c|}{ Actual } \\
\hline $\begin{array}{c}\text { Thickness } \\
/ \mathrm{mm}\end{array}$ & Fire condition & $\phi$ & $\begin{array}{c}\text { Corresponding } \\
\text { CO yield (g/g) }\end{array}$ & $\begin{array}{c}\text { mass } \\
\text { loss \% }\end{array}$ & $\begin{array}{c}\phi \text { (estimated } \\
\text { from mass } \\
\text { loss) }\end{array}$ & $\begin{array}{c}\text { Co } \\
\text { yield } \\
\text { (g/g) }\end{array}$ & $\begin{array}{c}\mathbf{O}_{2} \text { at peak } \\
\text { smoke \% }\end{array}$ \\
\hline 5 & well-vent'd & 0.61 & 0.02 & 95.7 & 0.54 & 0.07 & 12 \\
\hline 10 & under-vent'd & 1.22 & 0.08 & 57.6 & 0.67 & 0.04 & 12.8 \\
\hline 15 & under-vent'd & 1.83 & 0.17 & 30.4 & 0.54 & 0.04 & 14.4 \\
\hline
\end{tabular}




\section{Problems with smoke chamber for assessment of fire toxicity}

In the smoke chamber most of the toxic gas production should occur towards the end of the test, when the oxygen is most depleted, provided flaming combustion is maintained. However, it is this stage where it is most difficult to maintain flaming combustion. While the smoke chamber is well-suited to the measurement of visual obscuration, during the early stages of flaming, where the smoke can be quantified non-destructively, and is independent of the smoke layer height and distribution, using the smoke chamber to quantify toxic gas generation presents a number of problems.

The ventilation condition, or equivalence ratio, corresponding to the time when the products are generated is unknown, because an unknown fraction of the available oxygen will be accessible to the fire plume. Indeed, the circulation within the chamber will depend primarily on the heat release rate. When sampling a stratified fire effluent from a fixed point, such as a tube $20 \mathrm{~cm}$ below the chamber roof, this could be too low, so missing the main effluent plume altogether, or sample from its centre, without dilution. The use of a fan in some variants of the smoke density chamber standard, disrupts the burning process considerably, and result in recirculation of all the fire effluent through the flame zone. The act of sampling, and clean-up prior to gas analysis, of the fire effluent will change its composition - if the fire gases are sampled in real time, it may be possible to isolate individual fire stages, but then the effluent may change during analysis, or may not be returned to the chamber after analysis. If the analyte is returned to the chamber, it will usually have been filtered removing both the particulates and the gases absorbed onto them, to protect the analysers, while acid gases may have deposited onto the sampling line. Similarly, the high surface area of the chamber wall will promote deposition and disproportionately larger losses of certain analytes, such as hydrogen chloride $(\mathrm{HCl})$ or hydrogen bromide $(\mathrm{HBr})$. However, of greatest significance in quantification of the toxicity of fire effluents is that the most important under-ventilated conditions cannot be replicated.

It is most unfortunate that the widespread availability of the smoke density chamber in test laboratories has led to a practice where it is also used for assessment of fire toxicity, and has been adopted by the mass transport industries to quantify fire hazards on aircraft, trains and ships. In particular, the failure of the protocol to replicate under-ventilated flaming prevent it from being able to discriminate against those materials which produce very toxic effluents in a developed fire, from those producing less toxic effluents. Indeed, the current procedure adopted by the International Maritime Organisation [11], references ISO 19702, the standard relating to the use of FTIR for quantifying fire gas toxicity, and ISO 5659-2 [10], with no mention of how they should be combined.

\section{CONCLUSIONS}

Fire effluent toxicity is the largest killer, and greatest cause of injury in fires. For the purposes of fire hazard assessment, fire toxicity must be determined as a function of both the material and of fire conditions, particularly temperature and oxygen availability in the fire zone. The steady state tube furnace, and the fire propagation apparatus show acceptable agreement with large scale data over the range of fire conditions. The controlled atmosphere cone calorimeter and the smoke density chamber fail to replicate the high yields of asphyxiant gases, $\mathrm{CO}$ and $\mathrm{HCN}$, which occur in under-ventilated fires. This shows that they are unsuitable for assessment of toxic hazard of fire effluents. The non-dynamic tube furnace replicates the toxic product yields intermediate between well-ventilated and under-ventilated flaming, seemingly independent of the pyrolysis temperature. Only the SSTF and FPA are capable of generating data under controlled conditions, allowing the relationship between equivalence ratio and the toxic product yields to be investigated, in order to provide suitable inputs to engineering based assessment of toxic fire hazard.

The adoptions of the smoke density chamber for quantify fire toxicity in international shipping [11] and across European rail network is of great concern. The rejection by ISO of DIS 21489 due to its irreproducibility ignores the more serious issue. The apparent inability of the smoke density chamber to replicate under-ventilated flaming renders it unsuitable for the assessment of fire effluent toxicity. Typically, in well-ventilated flaming there would be 0.02 grams of carbon dioxide per gram of burning polymer and 0.002 grams of hydrogen cyanide per gram of nitrogen containing polymer while in underventilated flaming there would be typical yields of $0.2 \mathrm{~g} / \mathrm{g}$ of $\mathrm{CO}$ and $0.06 \mathrm{~g} / \mathrm{g}$ of HCN: increases by factors of 10 and 30. 


\section{REFERENCES}

[1] Hull, T. R., Quinn, R. E., Areri, I. G., and Purser, D. A. (2002). Combustion toxicity of fire retarded EVA. Polymer Degradation and Stability, 77: 235-242, http://dx.doi.org/10.1016/S01413910(02)00039-3

[2] Stec, A. A., Hull, T. R., Lebek, K., Purser, J. A., and Purser, D. A. (2008). The effect of temperature and ventilation condition on the toxic product yields from burning polymers. Fire and Materials, 32: 49-60, http://dx.doi.org/10.1002/fam.955

[3] ISO 19706:2007, Guidelines for assessing the fire threat to people.

[4] Markstein, G. H. (1988). Radiant emission and smoke points for laminar diffusion flames of fuel mixtures. Symposium (International) on Combustion, 21: 1107-1114.

[5] Alarie, Y. (2002). Toxicity of fire smoke. Critical reviews in toxicology, 32: 259-289, http://dx.doi.org/10.1080/20024091064246

[6] Purser, D. A. (2000). Toxic product yields and hazard assessment for fully enclosed design fires. Polymer International, 49: 1232-1255, http://dx.doi.org/10.1002/10970126(200010)49:10<1232::AID-PI543>3.0.CO;2-T

[7] Pitts, W. M. (1995). The global equivalence ratio concept and the formation mechanisms of carbon monoxide in enclosure fires. Progress in Energy and Combustion Science, 21: 197-237, http://dx.doi.org/10.1016/0360-1285(95)00004-2

[8] ISO 13571:2012 Life threat from fires - Guidance on the estimation of time available for escape using fire data.

[9] Hull, T. R., and Paul, K. T. (2007). Bench-scale assessment of combustion toxicity-A critical analysis of current protocols. Fire Safety Journal, 42: 340-365, http://dx.doi.org/10.1016/j.firesaf.2006.12.006

[10] ISO 5659-2:2012 Plastics - Smoke generation - Part 2: Determination of optical density by a single-chamber test

[11] 2010 Fire Test Procedure Code, Maritime Safety Committee, (MSC 87/26/Add.3) Annex 34, Part 2 Smoke and Toxicity Test, International Maritime Organisation, London.

[12] CEN/TS 45545-2:2009 Railway applications - Fire protection on railway vehicles - Part 2: Requirements for fire behaviour of materials and components.

[13] NF X 70-100, Analysis of pyrolysis and combustion gases. Tube furnace method. Part 1, Methods of analysis of gas generated by thermal degradation. Part 2, Method of thermal degradation using tube furnace.

[14] ISO 12136:2011, Reaction to fire tests - Measurement of material properties using a fire propagation apparatus.

[15] ISO/DIS 19700:2013 Controlled equivalence ratio method for the determination of hazardous components of fire effluents - the steady state tube furnace.

[16] ISO 5660-1:2002 Fire tests - Reaction to fire - Part 1: Rate of heat release from building products (cone calorimeter method)

[17] Stec, A. A., Hull, T. R., and Lebek, K. (2008). Characterisation of the steady state tube furnace (ISO TS 19700) for fire toxicity assessment. Polymer Degradation and Stability, 93: 2058-2065, http://dx.doi.org/10.1016/j.polymdegradstab.2008.02.020

[18] EN 2826:2011 Aerospace series - Burning behaviour of non metallic materials under the influence of radiating heat and flames - Determination of gas components in the smoke; ABD 0031 FireSmoke-Toxicity (FST) Test Specification (Airbus Industries); Boeing BSS 7239, Test method for toxic gas generation by materials on combustion. 
[19] Schartel, B., and Hull, T. R. (2007). Development of fire-retarded materials - interpretation of cone calorimeter data. Fire and Materials, 31: 327-354, http://dx.doi.org/10.1002/fam.949

[20] Babrauskas, V., Twilley, W. H., Janssens, M., and Yusa, S. (1992). Cone calorimeter for $\begin{array}{lllll}\text { controlled-atmosphere } & \text { studies. } & \text { Fire } & \text { 37-43, }\end{array}$ http://dx.doi.org/10.1002/fam.810160106

[21] Christy, M., Petrella, R., and Penkala, J. (1995). Controlled-atmosphere cone calorimeter. Fire and Polymers II: Materials and Tests for Hazard Prevention, 599, 498-517.

[22] Hietaniemi, J., Kallonen, R., and Mikkola, E. (1999). Burning characteristics of selected substances: Production of heat, smoke and chemical species. Fire and Materials, 23:171-185, http://dx.doi.org/10.1002/(SICI)1099-1018(199907/08)23:4<171::AID-FAM680>3.0.CO;2-C

[23] Tewarson, A., Generation of heat and chemical compounds in fires, SFPE handbook of fire protection engineering, Quincy MA. p. 3-82, 3rd ed., (2002).

[24] LeTallec, Y., Smith, D. A., Hunter, J., and Groenfeld, F.R., Evaluation of toxicants for materials used in military vehicles and equipment, 10th International Fire Science and Engineering Conference (Interflam) 631-637, (2004).

[25] ISO 13344:1996, Estimation of lethal toxic potency of fire effluents.

[26] Blomqvist, P., and Lonnermark, A. (2001). Characterization of the combustion products in largescale fire tests: Comparison of three experimental configurations. Fire and Materials, 25: 71-81, http://dx.doi.org/10.1002/fam.761

[27] Andersson, B., Markert, F., and Holmstedt, G. (2005). Combustion products generated by heteroorganic fuels on four different fire test scales. Fire Safety Journal, 40: 439-465, http://dx.doi.org/10.1016/j.firesaf.2005.03.002

[28] J Heitaniemi, R Kallonen and E Mikkola, Fires at Chemical Wharehouses - A cone calorimeter study on the burning characteristics and fire effluent composition of selected chemical compounds VTT Research Notes 1810, VTT, Espoo, Finland (1997).

[29] Babrauskas, V., Parker, W. J., Mulholland, G., and Twilley, W. H. (1994). The phi meter: A simple, fuel-independent instrument for monitoring combustion equivalence ratio. Review of Scientific Instruments, 65: 2367-2375, http://dx.doi.org/10.1063/1.1144690

[30] ISO 9705:1993, Fire tests - Full-scale room test for surface products

[31] Hull, T. R., Stec, A. A., Lebek, K., and Price, D. (2007). Factors affecting the combustion toxicity of polymeric materials. Polymer Degradation and Stability, 92: 2239-2246, http://dx.doi.org/10.1016/j.polymdegradstab.2007.03.032

[32] Blomqvist, P., Hertzberg, T. and Tuovinen, H. A small-scale controlled equivalence ratio tube furnace method - Experience of the method and link to large scale fires. Proceedings of the 11th International Interflam Conference Pp. 391-402. (2007) Interscience Communications Ltd., London.

[33] Purser D.A. and Purser, J.A., HCN yields and fate of fuel nitrogen for materials under different combustion conditions in the ISO 19700 tube furnace and large-scale fires, Fire Safety Science. (2009) 1117-1128. http://dx.doi.org/10.3801/IAFSS.FSS.9-1117

[34] Gomez, C., Janssens, M., and Zalkin, A., Measuring yields of toxic gases from materials during different stages of fire development, Proceedings of the 12th International Conference on Fire and Materials, Interscience Communications, (2011).

[35] Marquis, D. M., Guillaume, E., Camillo, A., Pavageau, M., Rogaume T., Usage of controlledatmosphere cone calorimeter to provide input data for toxicity modeling, Proceedings of the 12th International Conference on Fire and Materials, Interscience Communications, (2011). 
[36] David A. Purser, John A. Rowley, Peter J. Fardell and Mark Bensilum. Fully Enclosed Design Fires For Hazard Assessment In Relation To Yields Of Carbon Monoxide And Hydrogen Cyanide Interflam '99 Proceedings vol 2 pp 1163-1169, (1999).

[37] Kaczorek, K., MPhil Thesis, University of Central Lancashire (2009). 